\title{
Different forms of the Kadanoff-Baym equations in quantum statistical mechanics
}

\author{
A.S. Kondratyev ${ }^{1,2}$ and N. Shahid ${ }^{2}$ \\ ${ }^{1}$ Department of Physics, Herzen State Pedagogical University of Russia \\ 48 Moika River Embankment, St. Petersburg 191168, Russia \\ E-mail: kondrat6125@mail.ru \\ ${ }^{2}$ Abdus Salam School of Mathematical Sciences, GC University 68-B, New Muslim Town, Lahore, Pakistan \\ E-mail: nash_shhd@hotmail.com \\ Received January 10, 2011
}

\begin{abstract}
A new form of the Kadanoff-Baym equations for a system of interacting particles is offered on the basis of the retarded and advanced quantum Green's functions. The comparison of the traditional and the offered forms of the equations allows to analyze the question to what extent Landau-Silin kinetic equations for the neutral Fermi-liquid and for the electron liquid of normal metals take into account quickly varying in space and time disturbances.
\end{abstract}

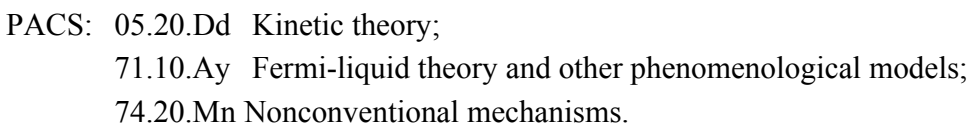

Keywords: Fermi-liquid, correlation functions, spectral function, quantum kinetic equations.

\section{Introduction}

The most successful microscopic theory which is capable of describing both the equilibrium and transport properties of many-particle systems in a comprehensive way is Kadanoff-Baym (KB) version of the Green's function formalism [1]. A tremendous variety of different applications of the KB method in recent years can be traced in [2-4]. It should be emphasized that the development of this method involves computational approach [5]. Special attention was devoted to further mathematical development of the KB method: the investigation of short-time kinetics and initial correlation in non-ideal quantum systems [6], Ward identity for nonequilibrium Fermi systems [7], solving the KB equations for inhomogeneous systems with application to atoms and molecules [8], derivation of the Landau-Silin kinetic equations for the neutral Fermi liquid [1] and electron liquid of normal metals [9-11], investigation of the mathematical properties of the spectral function for oneparticle states $[10,12]$, investigation of electrical conductivity in disordered metal alloys [13].

The successfulness of the phenomenological LandauSilin theory in predicting and describing a set of new phenomena, among them the zero sound in liquid ${ }^{3} \mathrm{He}$ and spin waves in nonferromagnetic metals, made this theory a sub- ject of investigation on the basis of strict microscopic theory. Experimental discovery of superfluidity of ${ }^{3} \mathrm{He}$ at very low temperature stimulated a second wave of attempts to widen the temperature range of validity of the LandauSilin kinetic equations. It was shown that these equations are valid in the case of finite temperature up to the terms linear in $\Gamma$ (see below) $[10,11]$. But the question of the validity of these equations for describing quickly varying disturbances remained open.

This paper is devoted to the investigation and further development of the KB method. We offer a new form of the exact $\mathrm{KB}$ equations in Wigner representation in terms of the correlation functions $g^{<}(\mathbf{p} \omega ; \mathbf{R} T)$ and $g^{>}(\mathbf{p} \omega ; \mathbf{R} T)$. This form turned to be more convenient for finding approximations for the correlation functions and the spectral function. Then we represent the KB theory in terms of commutator Green's functions $g_{a}(\mathbf{p} \omega ; \mathbf{R} T)$ and $g_{r}(\mathbf{p} \omega ; \mathbf{R} T)$ - advanced and retarded Green's functions, respectively.

The following section contains a concise presentation of the necessary formulas of the $\mathrm{KB}$ theory for nonequilibrium systems $[14,15]$. In Sec. 3 these formulas will be used for the derivation of a new form of the exact equations for the correlation functions. Sec. 4 containes the KB theory in terms of the advanced and retarded commutator Green's functions. Section 5 is devoted to the analysis of 
the question to what extent the Landau-Silin kinetic equations take into account quickly varying in space and time disturbances.

\section{Main formulas of the Kadanof-Baym formalism in Wigner representation}

We will start with the $\mathrm{KB}$ exact equations for the correlation functions $g^{<}$and $g^{>}$which after the transition to Wigner coordinates $\quad \mathbf{r}=\mathbf{r}_{1}-\mathbf{r}_{2}, \quad \mathbf{R}=\left(\mathbf{r}_{1}+\mathbf{r}_{2}\right) / 2$, $t=t_{1}-t_{2}, \quad T=\left(t_{1}+t_{2}\right) / 2$ and the Fourier transform with respect to the variables $\mathbf{r}$ and $t$ can be written in the form $[14,15]$ :

$$
\begin{aligned}
& \left\{\omega_{E}+\frac{i}{2} \Gamma, g^{<}\right\}-\left\{\sigma^{<}, \operatorname{Re} g+\frac{i}{2} a\right\}=0, \\
& \left\{g^{<}, \omega_{E}-\frac{i}{2} \Gamma\right\}-\left\{\operatorname{Re} g-\frac{i}{2} a, \sigma^{<}\right\}=0, \\
& \left\{\omega_{E}+\frac{i}{2} \Gamma, g^{>}\right\}-\left\{\sigma^{>}, \operatorname{Re} g+\frac{i}{2} a\right\}=0, \\
& \left\{g^{>}, \omega_{E}-\frac{i}{2} \Gamma\right\}-\left\{\operatorname{Re} g-\frac{i}{2} a, \sigma^{>}\right\}=0,
\end{aligned}
$$

where

$$
\{A, B\}=\exp \left[\frac{i}{2}\left(\frac{\partial_{A}}{\partial \omega} \frac{\partial_{B}}{\partial T}-\frac{\partial_{A}}{\partial T} \frac{\partial_{B}}{\partial \omega}-\nabla_{\mathbf{p}}^{A} \cdot \nabla_{\mathbf{R}}^{B}+\nabla_{\mathbf{R}}^{A} \cdot \nabla_{\mathbf{p}}^{B}\right)\right] A B .
$$

In these expressions

$$
\omega_{E}=\omega-E^{H F}(\mathbf{p} ; \mathbf{R} T)-\operatorname{Re} \sigma_{c}(\mathbf{p} \omega ; \mathbf{R} T),
$$

Spectral functions $a$ and $\Gamma$ are defined by the equalities

$$
\begin{aligned}
& a(\mathbf{p} \omega ; \mathbf{R} T)=g^{>}(\mathbf{p} \omega ; \mathbf{R} T) \mp g^{<}(\mathbf{p} \omega ; \mathbf{R} T), \\
& \Gamma(\mathbf{p} \omega ; \mathbf{R} T)=\sigma^{>}(\mathbf{p} \omega ; \mathbf{R} T) \mp \sigma^{<}(\mathbf{p} \omega ; \mathbf{R} T),
\end{aligned}
$$

The quantities $g$ and $\sigma_{c}$ are connected with $a$ and $\Gamma$ by the relations

$$
\begin{aligned}
& g(\mathbf{p} z ; \mathbf{R} T)=\int_{-\infty}^{+\infty} \frac{d \omega}{2 \pi} \frac{a(\mathbf{p} \omega ; \mathbf{R} T)}{z-\omega} \\
& \sigma_{A}(\mathbf{p} z ; \mathbf{R} T)=\int_{-\infty}^{+\infty} \frac{d \omega}{2 \pi} \frac{\Gamma(\mathbf{p} \omega ; \mathbf{R} T)}{z-\omega}
\end{aligned}
$$

The upper sign in (7) and (8) refers to Bose-Einstein particles and the lower sign refers to Fermi-Dirac particles. The quantity $E^{H F}(\mathbf{p} ; \mathbf{R} T)$ is a one-particle energy in the Hartree-Fock approximation including the external field $U(\mathbf{R} T)$, and all other notations coincide with those in [1].

The exact equation for the spectral function $a(\mathbf{p} \omega ; \mathbf{R} T)$ is obtained with the help of (1) and (3) and looks like

$$
\left\{\omega_{E}+\frac{i}{2}, a\right\}-\left\{\Gamma, \operatorname{Re} g+\frac{i}{2} a\right\}=0,
$$

If we expand the exponents in the Eqs. (1)-(4) into Taylor series and save the terms up to the first derivatives, we get the generalized $\mathrm{KB}$ quantum kinetic equations. For example, the equation for the function $g^{<}$looks like

$$
\left[\omega_{E}, g^{<}\right]_{P}-\left[\sigma^{<}, \operatorname{Re} g\right]_{P}=\sigma^{<} g^{>}-\sigma^{>} g^{<}
$$

where we consider that all the quantities in (1)-(4) commute. The generalized Poisson bracket in (11) is defined by the expression

$[A, B]_{P}=\frac{\partial A}{\partial \omega} \frac{\partial B}{\partial T}-\frac{\partial A}{\partial T} \frac{\partial B}{\partial \omega}-\nabla_{\mathbf{p}} A \cdot \nabla_{\mathbf{R}} B+\nabla_{\mathbf{R}} A \cdot \nabla_{\mathbf{p}} B$

The approximate equation for the function $a(\mathbf{p} \omega ; \mathbf{R} T)$ corresponding to the equations (11) and (12) has the form

$$
\left[\omega_{E}, a\right]_{P}-[\Gamma, \operatorname{Reg}]_{P}=0,
$$

Equations (1)-(4) and (10) can be used for the description of all kinds of transport phenomena in different approximations. Nevertheless, the structure of these equations is not convenient for the investigation of the approximations.

\section{A new form of the exact equations for the correlation functions and the spectral functions}

The Fourier transforms of the correlation functions contain the explicit factors of $i$ and $\pm i$. For example [1],

$$
g^{<}(\mathbf{p} \omega ; \mathbf{R} T)=\int d t d \mathbf{r} \exp (-i \mathbf{p} \cdot \mathbf{r}+i \omega t)\left[ \pm i g^{<}(\mathbf{r} t ; \mathbf{R} T)\right]
$$

These factors make the functions $g^{<}(\mathbf{p} \omega ; \mathbf{R} T)$ or $g^{>}(\mathbf{p} \omega ; \mathbf{R} T)$ real nonnegative quantities [1]. It allows to give another representation for the Eqs. (1)-(4) and (10) which reveals certain properties of quantum systems and of the different approximations for the exact equations.

We use the Euler formula for a real $A$ and $B$ :

$$
\exp [i D] A B=\cos [D] A B+i \sin [D] A B,
$$

where the operator $D$ is defined by

$$
[D] A B=\frac{1}{2}\left(\frac{\partial_{A}}{\partial \omega} \frac{\partial_{B}}{\partial T}-\frac{\partial_{A}}{\partial T} \frac{\partial_{B}}{\partial \omega}-\nabla_{\mathbf{p}}^{A} \cdot \nabla_{\mathbf{R}}^{B}+\nabla_{\mathbf{R}}^{A} \cdot \nabla_{\mathbf{p}}^{B}\right) A B .
$$

The operators $\cos [D]$ and $\sin [D]$ are understood in the sense of their Taylor series.

Formula (15) gives the possibility to get a new representation for the Eqs. (1)-(4) and (10) by separating real and imaginary parts in these equations. For the spectral function $a(\mathbf{p} \omega ; \mathbf{R} T)$ we get instead of (10) two equations:

$$
\begin{aligned}
& \cos [D]\left(\omega_{E} a-\Gamma \operatorname{Re} g\right)=0, \\
& \sin [D]\left(\omega_{E} a-\Gamma \operatorname{Re} g\right)=0 .
\end{aligned}
$$


The expansion of $\cos [D]$ in the Eq. (17) leads to the following expression for the equilibrium value of the spectral function:

$$
a=\frac{\Gamma \operatorname{Re} g}{\omega_{E}}=\frac{\Gamma}{\omega_{E}^{2}+\Gamma^{2} / 4},
$$

which coincides with the corresponding expression in [1]. Here the quantities $\omega_{E}, g$ and $\Gamma$ are equal to their equilibrium values. The expansion of $\sin [D]$ in (18) leads to different possible approximate equations for the nonequilibrium spectral function. The simplest approximation, when only first derivatives are taken into account, coincides with the Eq. (13).We should emphasize that the expansions of the Eqs. (17) and (18) contain only the derivatives of even (for (17)) and odd (for (18)) order.

Another situation is valid for the equations for the correlation functions $g^{<}$and $g^{>}$. For example, the equations for the function $g^{<}$have the forms:

$$
\begin{aligned}
& \cos [D]\left(\omega_{E} g^{<}-\sigma^{<} \operatorname{Re} g\right)=\frac{1}{2} \sin [D]\left(\sigma^{>} g^{<}-\sigma^{<} g^{>}\right), \\
& \sin [D]\left(\omega_{E} g^{<}-\sigma^{<} \operatorname{Re} g\right)=\frac{1}{2} \cos [D]\left(\sigma^{<} g^{>}-\sigma^{>} g^{<}\right),
\end{aligned}
$$

because

$$
\sigma^{<} a-\Gamma g^{<}=\sigma^{<} g^{>}-\sigma^{>} g^{<} .
$$

The representation based on the formula (15) was used in $[16,17]$ for the description of the systems of noninteracting particles in an external field. As it is shown in [17] the equation of motion in the form of quantum Liouville equation for the Wigner function generates two phase space equations which determine the Moyal function. One of them contains only odd derivatives and another one contains only even derivatives. For an arbitrary potentials both these equations are of infinite order and exact analytic solution cannot be presented. This property for the systems of interacting particles is valid, as we have shown, only for the equations for the spectral function. The Eqs. (20) and (21) for the correlation functions, on the contrary, contain the derivatives both of odd and even orders. If we neglect the quantities $\sigma^{<}$and $\sigma^{>}$(what means the case of the Hartree-Fock approximation for the interaction between particles), the Eqs. (20) and (21) contain the derivatives only of the even (for (20)) or for odd (for (21)) order.

\section{Exact equations for the retarded and advanced commutator Green's functions}

Another form of the exact Eqs. (1)-(4) can be found in terms of the retarded $g_{r}$ and advanced $g_{a}$ Green's functions which can be expressed through the KB correlation functions $g^{<}$and $g^{>}$:

$$
g_{r}\left(1,1^{\prime}\right)=\left(g^{>}\left(1,1^{\prime}\right)+g^{<}\left(1,1^{\prime}\right)\right) \Theta\left(t_{1}-t_{1^{\prime}}\right)
$$

$$
g_{a}\left(1,1^{\prime}\right)=-\left(g^{>}\left(1,1^{\prime}\right)+g^{<}\left(1,1^{\prime}\right)\right) \Theta\left(t_{1^{\prime}}-t_{1}\right),
$$

Here usual KB notation $1=\left(\mathbf{r}_{1}, t_{1}\right)$ is used and $\Theta(x)$ is a Heviside's step function. Here on the contrary to (14) it is convenient to define the Fourier transforms of these functions in Wigner coordinates without the factor $i$ :

$g_{r, a}(\mathbf{p} \omega ; \mathbf{R} T)=\int d t d \mathbf{r} \exp (-i \mathbf{p} \cdot \mathbf{r}+i \omega t) g_{r, a}(\mathbf{r} t ; \mathbf{R} T)$

The functions $\sigma_{r, a}$ are introduced in the same way as the functions $g_{r, a}$. The KB spectral functions $a$ and $\Gamma$ can be expressed in the following way:

$$
\begin{aligned}
& a(\mathbf{p} \omega ; \mathbf{R} T)=i\left[g_{r}(\mathbf{p} \omega ; \mathbf{R} T)-g_{a}(\mathbf{p} \omega ; \mathbf{R} T)\right], \\
& \Gamma(\mathbf{p} \omega ; \mathbf{R} T)=i\left[\sigma_{r}(\mathbf{p} \omega ; \mathbf{R} T)-\sigma_{a}(\mathbf{p} \omega ; \mathbf{R} T)\right] .
\end{aligned}
$$

It was shown in [18] that a typical term in the KB theory

$$
D\left(1,1^{\prime}\right)=\int_{t_{0}}^{t_{0}-i \beta} d 2 A(1,2) B\left(2,1^{\prime}\right)
$$

after the transition $t_{0} \rightarrow-\infty$ and the Fourier transform in Wigner coordinates can be presented in the following way. For example, for $D^{<}$

$$
\begin{gathered}
D^{<}(\mathbf{p} \omega ; \mathbf{R} T)=\left\{A_{r}(\mathbf{p} \omega ; \mathbf{R} T), B^{<}(\mathbf{p} \omega ; \mathbf{R} T)\right\}+ \\
+\left\{A^{<}(\mathbf{p} \omega ; \mathbf{R} T), B_{a}(\mathbf{p} \omega ; \mathbf{R} T)\right\}
\end{gathered}
$$

where $\{A, B\}$ has the same sense as in (5). It can be shown by direct calculation that

$$
D_{r, a}(\mathbf{p} \omega ; \mathbf{R} T)=\left\{A_{r, a}(\mathbf{p} \omega ; \mathbf{R} T), B_{r, a}(\mathbf{p} \omega ; \mathbf{R} T)\right\} .
$$

In terms of the introduced functions the exact Eqs. (1)-(4) can be written in the following way. For the function $g^{<}$ we get:

$$
\begin{gathered}
\left\{\omega-E^{H F}(\mathbf{p} ; \mathbf{R} T)-\sigma_{r}(\mathbf{p} \omega ; \mathbf{R} T), g^{<}(\mathbf{p} \omega ; \mathbf{R} T)\right\}= \\
=\left\{\sigma^{<}(\mathbf{p} \omega ; \mathbf{R} T), g_{a}(\mathbf{p} \omega ; \mathbf{R} T)\right\}, \\
\left\{g^{<}(\mathbf{p} \omega ; \mathbf{R} T), \omega-E^{H F}(\mathbf{p} ; \mathbf{R} T)-\sigma_{a}(\mathbf{p} \omega ; \mathbf{R} T)\right\}= \\
=\left\{g_{r}(\mathbf{p} \omega ; \mathbf{R} T), \sigma^{<}(\mathbf{p} \omega ; \mathbf{R} T)\right\} .
\end{gathered}
$$

The equations for the function $g^{>}$are obtained by changing the sign «less» < for the sign «more» $>$ in the Eqs. (31) and (32).

The functions $\operatorname{Re} g$ and $\operatorname{Re} \sigma_{c}$ in the Eqs. (1)-(4) and the functions $g_{r, a}$ and $\sigma_{r, a}$ in the Eqs. (31) and (32) are connected by the relations:

$$
\begin{array}{r}
\operatorname{Re} g(\mathbf{p} \omega ; \mathbf{R} T)=\frac{1}{2}\left(\left(g_{r}(\mathbf{p} \omega ; \mathbf{R} T)+g_{a}(\mathbf{p} \omega ; \mathbf{R} T)\right),\right. \\
\operatorname{Re} \sigma_{c}(\mathbf{p} \omega ; \mathbf{R} T)=\frac{1}{2}\left(\left(\sigma_{r}(\mathbf{p} \omega ; \mathbf{R} T)+\sigma_{a}(\mathbf{p} \omega ; \mathbf{R} T)\right) .\right.
\end{array}
$$


The equivalence of the Eqs. (1)-(4) and the Eqs. (31) and (32) can be shown with the help of (29). The exact equations for the functions $g_{r, a}$ can be obtained with the help of (30):

$$
\begin{gathered}
\left\{\omega-E^{H F}(\mathbf{p} ; \mathbf{R} T), g_{r, a}(\mathbf{p} \omega ; \mathbf{R} T)\right\}= \\
=1+\left\{\sigma_{r, a}(\mathbf{p} \omega ; \mathbf{R} T), g_{r, a}(\mathbf{p} \omega ; \mathbf{R} T)\right\} \\
\left\{g_{r, a}(\mathbf{p} \omega ; \mathbf{R} T), \omega-E^{H F}(\mathbf{p} ; \mathbf{R} T)\right\}= \\
=1+\left\{g_{r, a}(\mathbf{p} \omega ; \mathbf{R} T), \sigma_{r, a}(\mathbf{p} \omega ; \mathbf{R} T)\right\} .
\end{gathered}
$$

The relations (33) and (34) allow to bring these equations to the following form:

$$
\begin{aligned}
& \left\{\omega_{E}+\frac{i}{2} \Gamma, g_{r}\right\}=1, \\
& \left\{g_{r}, \omega_{E}+\frac{i}{2} \Gamma\right\}=1, \\
& \left\{\omega_{E}+\frac{i}{2} \Gamma, g_{a}\right\}=1, \\
& \left\{g_{a}, \omega_{E}+\frac{i}{2} \Gamma\right\}=1 .
\end{aligned}
$$

The system of the Eqs. (37)-(40) is equivalent to the system (1)-(4). But the system (37)-(40) is more convenient for finding certain approximations for the spectral function $a(\mathbf{p} \omega ; \mathbf{R} T)$. It follows from the Eqs. (37) and (38) that the left sides of these equations are equal:

$$
\left\{\omega_{E}+\frac{i}{2} \Gamma, g_{r}\right\}=\left\{g_{r}, \omega_{E}+\frac{i}{2} \Gamma\right\} .
$$

The equality of the even powers of the expansions of the exponents in (41) follows automatically. The equality of odd powers of the expansions follows, if the function $g_{r}$ has the argument:

$$
g_{r}(\mathbf{p} \omega ; \mathbf{R} T)=f\left(\omega_{E}+\frac{i}{2} \Gamma(\mathbf{p} \omega ; \mathbf{R} T)\right)
$$

The same considerations lead to the following form of the advanced function $g_{a}$ :

$$
g_{a}(\mathbf{p} \omega ; \mathbf{R} T)=f\left(\omega_{E}-\frac{i}{2} \Gamma(\mathbf{p} \omega ; \mathbf{R} T)\right)
$$

It is easy to prove that the function $f$ in the expressions (42) and (43) is the same because only in this case we get a proper expression for the spectral function $a$ equivalent to (19).

Equations (37) and (39) lead to the following approximate equations which take into account only the derivatives of the second order:

$$
\left(\omega_{E}-\frac{i}{2} \Gamma\right) g_{a}-\frac{1}{8}\left[\omega_{E}-\frac{i}{2} \Gamma, g_{a}\right]_{2}=1
$$

where $[A, B]_{2}$ is defined by the expression:

$$
\begin{gathered}
{[A, B]_{2}=\left[\frac{\partial A}{\partial \omega}, \frac{\partial B}{\partial T}\right]_{P}-\left[\frac{\partial A}{\partial T}, \frac{\partial B}{\partial \omega}\right]_{P}-} \\
-\left[\nabla_{\mathbf{p}} A, \nabla_{\mathbf{R}} B\right]_{P}+\left[\nabla_{\mathbf{R}} A, \nabla_{\mathbf{p}} B\right]_{P} .
\end{gathered}
$$

The Eqs. (44) and (45) can be solved by iterations what allows to analyze the question to what extent Landau-Silin kinetic equations take into account quick disturbances in space and time.

\section{Quickly varying disturbances in Landau-Silin equations}

Solving by iterations the Eqs. (44) and (45) and using the formulas (26) and (33), after some calculations we come to the following expressions for the spectral function $a(\mathbf{p} \omega ; \mathbf{R} T)$ and $\operatorname{Re} g(\mathbf{p} \omega ; \mathbf{R} T)$, which are valid with the precision up to the second derivatives:

$$
\begin{gathered}
a=a_{0}+\frac{1}{8} \operatorname{Re} g_{0}\left[\omega_{E}, a\right]_{2}-\frac{1}{8} \operatorname{Re} g_{0}\left[\Gamma, \operatorname{Re}_{0}\right]_{2}+ \\
+\frac{1}{8} a_{0}\left[\omega_{E}, \operatorname{Re} g_{0}\right]_{2}+\frac{1}{32} a_{0}\left[\Gamma, a_{0}\right]_{2} \\
\operatorname{Reg}=\operatorname{Re}_{0}+\frac{1}{8} \operatorname{Re} g_{0}\left[\omega_{E}, \operatorname{Re} g_{0}\right]_{2}+\frac{1}{32} \operatorname{Re} g_{0}\left[\Gamma, a_{0}\right]_{2}- \\
-\frac{1}{32} \Gamma\left[\omega_{E}, a_{0}\right]_{2}+\frac{1}{32} \Gamma\left[\Gamma, \operatorname{Re} g_{0}\right]_{2},
\end{gathered}
$$

where

$$
a_{0}=\frac{\Gamma}{\omega_{E}^{2}+\Gamma^{2} / 4}, \quad \operatorname{Reg} g_{0}=\frac{\omega_{E}}{\omega_{E}^{2}+\Gamma^{2} / 4}
$$

According to (46) the expressions (47) and (48) contain only the derivatives of the second order, the first derivatives are absent. It was proved in $[10,11]$ that the kinetic equations of the phenomenological theory are valid in the case of finite temperature up to the terms linear in $\Gamma$. Thus, analyzing the kinetic equations it is lawful to take into account only the independent of $\Gamma$ and linear in $\Gamma$ terms in the expressions (47) and (48). Keeping in mind the formulas (49) which satisfy the Eq. (13), we should restrict the expressions (47) and (48) by the following terms:

$$
\begin{gathered}
a=a_{0}+\frac{1}{8}\left[\omega_{E}, \frac{\Gamma}{\omega_{E}^{2}}\right]_{2}-\frac{1}{8 \omega_{E}}\left[\Gamma, \frac{1}{\omega_{E}}\right]_{2}+\frac{\Gamma}{8 \omega_{E}^{2}}\left[\omega_{E}, \frac{1}{\omega_{E}}\right]_{2}, \\
\operatorname{Reg}=\operatorname{Reg}_{0}+\frac{1}{8 \omega_{E}}\left[\omega_{E}, \frac{1}{\omega_{E}}\right]_{2}
\end{gathered}
$$


It is not difficult to prove that the expressions (50) and (51) satisfy the Eq. (13) for the spectral function. Indeed, if we substitute (50) and (51) into the Eq. (13) and take into account the properties of the generalized Poisson bracket

$$
\begin{gathered}
{[A, \varphi(A)]_{P}=0,\left(\text { but }[A, \varphi(A)]_{2} \neq 0\right),} \\
{\left[A, \frac{B}{A^{2}}\right]_{P}+\left[\frac{1}{A}, B\right]_{P}=0,}
\end{gathered}
$$

we get an identity. The obtained result means that the quantities $g^{<}$and $\sigma^{<}$satisfy the generalized $\mathrm{KB}$ quantum kinetic Eq. (11) in the collisionless approximation. It can be shown just in the same way as in $[10,11]$ by substitution to (11) the quantities $g^{<}$and $\sigma^{<}$expressed through the spectral functions $a$ and $\Gamma$ and taking into account the properties (52) and (53). Thus, all the additional terms which could appear in the Landau-Silin kinetic equations due to quickly varying in space and time disturbances, cancel each other provided the disturbances are restricted by the derivatives of the second order and only independent of $\Gamma$ and linear in $\Gamma$ terms are taken into account.

\section{Conclusion}

The presented analysis helps to understand on the qualitative level the validity of different models of Fermiliquid approach for to the description of magnetosize effects in metals and alloys at low but finite temperature [19], although the complete theory of a system in a strong magnetic field is much more complicated. The possibility of an adequate treatment of high frequency effects in the frame of quantum kinetic equation makes reasonable and reliable the results presented in [19] for rather simple phenomenological models. At the same time the forms (17) and (18) for the exact equations can be more convenient for different applications as it occurred with corresponding equations $[16,17]$.
1. L.P. Kadanoff and G. Baym, Quantum Statistical Mechanics, Benjamin, New York (1962); Perseus Books, Cambridge, MA (1989).

2. Progress in Nonequilibrium Green's Functions, M. Bonitz (ed.), World Scientific Publ., Singapore (2000).

3. Progress in Nonequilibrium Green's Functions II, M. Bonitz and D. Semkat (eds.), World Scientific Publ., Singapore (2003).

4. D. Zubarev, V. Morozov, and G. Röpke, Statistical Mechanics of Non-equilibrium Processes, v. II, Academic Verlag (1996).

5. Introduction to Computational Methods in Many-Body Physics, M. Bonitz and D. Semkat (eds.), Rinton Press, Paramus (2006).

6. D. Kremp, D. Semkat, and Th. Bornath, Condens. Matter. Phys. 9, N3 (47), 431 (2006).

7. B. Velicky, A. Karlova, and V. Spicka, Phys. Rev. B77, 041201 (R) (2008).

8. N.E. Dahlem and R. van Leenwen, Phys. Rev. Lett. 98, 153004 (2007).

9. S.B. Anokhin and A.S. Kondratyev, J. Exp. Theor. Phys. 55, 1356 (1968) [Sov. Phys. JEPT 28, 710 (1969)].

10. M. Arshad, A.S. Kondratyev, and I. Siddique, Phys. Rev. B76, 054306 (2007).

11. A.S. Kondratyev and I. Siddique, Fiz. Niz. Temp. 34, 179 (2008) [Low Temp. Phys. 34, 137 (2008)].

12. R. Zeinstra, A. Alenitsyn, and M. Arshad, J. Math. Sciences 150, 1799 (2008).

13. L.Yu. Zakharov and Yu.M. Ivanchenko, Fiz. Niz. Temp. 4, 41 (1978) [Sov. J. Low Temp. Phys. 4, 19 (1978)].

14. A.S. Kondratyev and A.E. Kutchma, Theor. Math. Phys. 24, 278 (1975).

15. A.S. Kondratyev and A.E. Kutchma, Lectures in the Quantum Liquids Theory, Leningrad University Publ. House (1989).

16. S.R. de Groot and L.G. Suttorp, Foundations of Electrodynamics, North-Holland Publ. Comp., Amsterdam (1972).

17. W.P. Schleich, Quantum Optics in Phase Space, WileyVCH (2001).

18. D.C. Langreth and J.W. Wilkins, Phys. Rev. B6, 3189 (1972).

19. A.S. Kondratyev, S.S. Nedorezov, and V.G.Peschanskii, Fiz. Metal. Metalloved. 5, 5 (1991). 